\title{
Dichotomies, trichotomies, quadrichotomies dans la « théorie traductologique » de J .-R. Ladmiral ${ }^{1}$
}

\author{
Georgiana LUNGU-BADEA
}

Université de l'Ouest, Timișoara

Roumanie

\begin{abstract}
Résumé: Dans cet article nous présentons les lignes directrices de la théorie traductologique ladmiralienne: les «théorèmes pour la traduction », la dichotomie sourciers ciblistes, le triangle méthodologique et le quatrain traductologique. Ce sont des repères essentiels qui ont marqué la recherche en traductologie internationale.
\end{abstract}

Mots-clés : traductologie, théorèmes, sourciers-ciblistes, théorie traductologique

\begin{abstract}
In this article, we present the guiding lines of Ladmiral's translation theory: «theorems for translation», dichotomy sourciers - ciblistes, source - target, the methodological triangle and the translation theory rectangle. These are the essential landmarks of international translation theory research.
\end{abstract}

Keywords : translation studies, theorems, source- target, translation theory.

Il nous est difficile de présenter ici toute la richesse du parcours du Professeur Jean-René Ladmiral dans ses multiples facettes. Je me contenterai donc d'indiquer quelques éléments et repères qui ont particulièrement retenu l'attention de l'Université de l'Ouest de Timiúoara qui l'accueille et l'honore solennellement aujourd'hui. De la carrière de J ean-René Ladmiral, marquée par l'excellence dès le début de ses études, nous retenons le D.E.S. de philosophie: Heidegger lecteur de Kant, sous la direction de Paul Ricour, à la Faculté des Lettres de Nanterre, ensuite sa

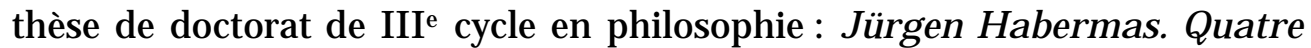
essais sur la raison, la pratique et la technique: traduction et commentaire (1974), toujours sous la direction du Professeur Ricœur à l'Université de Paris-X-Nanterre, son Habilitation à diriger des recherches obtenue en 1995, après avoir soutenu sa thèse de doctorat d'État. Cette

${ }^{1}$ Nous présentons ici la forme remaniée de l'Éloge de Monsieur Jean-René Ladmiral, lu dans la séance de remise du diplôme et des insignes de docteur honoris causa par l'Université de l'Ouest de Timisoara, le 25 mars 2010. 
Habilitation, intitulée La traductologie : de la linguistique à la philosophie, regroupe, outre le rapport de synthèse, un ouvrage et deux volumes d'articles.

Grâce à sa thèse de doctorat en philosophie sur Jürgen Habermas (1974), et grâce à ses traductions d'Habermas, Jean-René Ladmiral fit découvrir ce philosophe quasi méconnu des Français à l'époque. Les exégèses précises, les nombreuses interprétations, les commentaires très riches, les études philosophiques et les traductions non seulement d'Habermas, mais également d'Adorno, de Fromm, de Kant ou d'Heidegger imposèrent Jean-René Ladmiral comme spécialiste incontesté dans le domaine de la philosophie allemande moderne et contemporaine.

Outre les traductions philosophiques, Jean-René Ladmiral écrivit et fit publier cinq ouvrages devenus des références pour tous les traducteurs et traductologues d'aujourd'hui et d'innombrables articles et études sur la traduction et la traductologie.

Germaniste, Jean-René Ladmiral a longuement travaillé sur l'interculturalité franco-allemande, sujet sur lequel il a publié un livre en collaboration avec le psycho-sociologue Edmond Marc Lipiansky, La communication interculturelle (1989), ouvrage pour lequel il ne cesse d'être consulté et sollicité. L’un des mérites de ce livre est de " s'interroger sur les capacités des sociétés à intégrer des modèles culturels mais aussi sur leur curieuse incapacité à échapper aux formes des sociétés multicommunautaires basées sur le paradigme américain. » (Nègre 1994, 183).

La didactique des langues et, particulièrement, la didactique de la traduction professionnelle ont été développées à l'ISIT de Paris (Institut Supérieur de Management et de Communication Interculturels, longtemps Institut Supérieur d'Interprétation et de Traduction). Une série importante d'ouvrages, d'articles et d'études publiés par le Professeur Ladmiral ont ainsi comme thèmes de recherche l'enseignement de la traduction et la formation des traducteurs dans les écoles de traduction. L'approche historique de la traduction y est effectuée dans la même perspective dichotomique qui nourrit les fondements de sa théorie de traduction.

Après avoir été vice-président de l'IRSOL (Institut de Recherches Sociolinguistiques de Paris), Jean-René Ladmiral a fondé et dirigé le CERT (Centre d'Études et de Recherches en Traduction) à l'Université de Paris-XNanterre. Il est à présent directeur du CRATIL (Centre de Recherche Appliquée sur la Traduction, l'Interprétation et le Langage) au sein de l'ISIT de Paris.

Professeur de linguistique et de philosophie à l'Université Paris-XNanterre dès 1968 et professeur de traductologie à l'ISIT de Paris, JeanRené Ladmiral réussit de façon novatrice à prodiguer un enseignement de la traduction original et à assurer la formation des traducteurs. En 
4. Hommages aux traducteurs et aux traductologues/ Hommage to translators and traductologists/ Würdigungen von Übersetzern und Übersetzungstheoretikern/ Homenajes a los traductores/

conséquence, il fut chargé de nombreuses missions hors de France (Italie, Grèce, Algérie, Grande-Bretagne, Belgique, Suisse, Canada, Maroc ...) et il fut professeur invité de plusieurs écoles de traduction et d'interprétation prestigieuses, telle l'École de Traduction et d'Interprétation (E.T.I.) de l'Université de Genève ou les universités de Saarbrücken, Oran, Alger, Beyrouth, Genève, Tartu, etc.

En tant que traductologue et (co)fondateur de la science qu'on appelle aujourd'hui «traductologie », plusieurs colloques et manifestations scientifiques lui furent consacrés, dont le Congrès Traduire "Traduire ». Autour des travaux de Jean René Ladmiral (ISIT Paris, 2004), etc. L'impact de ses idées sur les traducteurs, traductologues et philosophes est extraordinaire (cf. Anna Makaryeva 2004, 103). D’une modestie louable, mais certainement excessive, Jean-René Ladmiral reconnaît qu' « [...] il arrive qu'on m'attribue la paternité de ce concept [traductologie]; mais en histoire des sciences, comme on sait, une idée neuve vient en général à l'esprit de deux ou trois chercheurs à peu près en même temps, dans la mesure où elle relève du paradigme épistémologique contemporain de ces derniers; et il en va de même pour les sciences humaines et, en l'occurrence, du concept de traductologie.» («Dichotomies traductologiques » 2004, 27).

Le traductologue Jean-René Ladmiral est connu du public roumain surtout pour son livre fondamental de traductologie Traduire : théorèmes pour la traduction (1979, réédité en 1994, 2002, 2010), traduit en plusieurs langues (allemand, anglais, grec, etc.). La théorie ladmiralienne en traduction jouit de l'estime des traductologues et universitaires et a donné lieu à de nombreuses exégèses. Jean-René Ladmiral, comme d'autres traductologues et philosophes français et étrangers (Antoine Berman, Henri Meschonnic, Paul Ricœur, Georges Mounin, Jean-Paul Vinay, Jean Darbelnet ou Georges-Arthur Goldschmidt), situe la traduction au cœur de la philosophie du langage et aux confins de la philosophie, de la linguistique, de la littérature et de la psychologie et témoigne ainsi de la " manière française » de traduire et de concevoir la traduction.

Jean-René Ladmiral s’inscrit dans la lignée intellectuelle d’illustres auteurs cités tout à l'heure, lignée qui défend les valeurs de métissage culturel, d'interdisciplinarité, d'universalisme, de globalisation de la science. Rappelons certaines de ces valeurs que le Professeur Ladmiral n'a cessé de promouvoir.

Selon Jean-René Ladmiral, la traductologie n'est pas seulement un espace philosophique, littéraire, mais aussi une praxis, un enjeu de développement économique et social ; la traductologie n'est pas non plus seulement une philosophie, mais également une "science clinique » au service des traducteurs. 
Revenons à la question fondamentale... L'enjeu de la traductologie a été dans un premier temps d'institutionnaliser et même d'organiser la pratique et la réflexion imposée par l'analyse de la pratique. Ceci l'a amené à identifier le «quatrain traductologique »: traductologie descriptive, prescriptive, inductive et productive ( 1997) qu'il reconsidèrera plus tard et présentera comme les « quatre modes d'approches de la traduction qui tendent à être contemporains, et donc concurrents » (« Dichotomies traductologiques » 2004, 34). Même s'il reste des choses à faire, ce programme de la traductologie a joui d'un grand succès. Aujourd'hui, la traductologie est vue comme une richesse. Elle se traduit par la promotion de la diversité des expressions et du dialogue. C'est aujourd'hui un moment privilégié pour rappeler l'engagement de la traductologie en faveur de la diversité culturelle et linguistique.

$\mathrm{La}$ «théorie de la traduction » de Jean-René Ladmiral constitue un cadre profondément original, en continuelle construction et en constants ajustements. Il y revient toujours dans ses œuvres et ses cours donnés à l'Université Paris X Nanterre, à l'ISIT de Paris ou depuis cette année à l'université de Paris-Sorbonne. Quelle est cette théorie?

- Une théorie qui interroge les fausses évidences (la traductologie est-elle une discipline autonome ou une sous-discipline? Une science ?), qui inverse les points de vue et prend donc pour « objet spécifique l'activité même de traduire, en amont de ce qui deviendra le texte-cible d' "une traduction"». Au lieu de travailler à une description linguistique », le Professeur Ladmiral s'engage «dans la démarche réflexive d'une traductologie productive » (« Dichotomies traductologiques » 2004, 35), en concevant notamment la traduction comme étant essentiellement un phénomène binaire (en s'inspirant de la méthode platonicienne, « Dichotomies traductologiques » 2004, 26).

- Une théorie foncièrement moderne, au sens où elle décrit moins des produits traductionnels que les opérations et les stratégies qui y conduisent.

- Une théorie résolument interdisciplinaire, qui en appelle tout à la fois à la linguistique (car « s'agissant de la traduction, il est communément admis que c'est l'affaire des linguistes ", in "Dichotomies traductologiques " 2004, 26), à la philosophie, à la psychologie cognitive, à la logique, à l'épistémologie et à l'informatique.

Grâce aux théorèmes de traduction et au célèbre clivage sourciersciblistes, qui ont marqué le monde traductologique international, le Professeur Jean-René Ladmiral donne l'image à la fois simple, complète et cohérente des deux plus grandes tendances traductionnelles de l'histoire et obtient ainsi un appareil métatraductologique qui lui permet de systématiser les recherches traductologiques contemporaines et de les regrouper selon l'objet, la méthode et la méthodologie de la recherche. La 
4. Hommages aux traducteurs et aux traductologues/ Hommage to translators and traductologists/ Würdigungen von Übersetzern und Übersetzungstheoretikern/ Homenajes a los traductores/

recherche de Jean-René Ladmiral couvre de la sorte un large champ d'aspects et de problématiques. De la traductologie à la philosophie du langage, l'œuvre de Jean-René Ladmiral a une valeur intrinsèque indéniable et une valeur didactique tellement profonde qu'elle se retrouve à l'origine de nombreuses recherches qui s'en réclament implicitement ou explicitement. Ses études épistémologiques d'une signification inestimable pour l'étude de la traduction et la compréhension de la traductologie contribuent à la nécessaire mutation des mentalités traductionnelle et traductologique.

Ses activités variées dans les domaines linguistique, philosophique, traductionnel, didactique et traductologique sont étroitement liées à la revue Le Journal du traducteur où il a publié de nombreux articles et études, aux revues (indexées par ISI) Meta: Le Journal des traducteurs, TTR, SEPTET, Palimpsestes, Traduire. Revue française de la traduction.

L'un des pères de la traductologie contemporaine, Jean-René Ladmiral est également membre de nombreux comités d'honneur et scientifiques internationaux (revues, colloques, congrès, conférences). Il est également membre du comité d'honneur de la revue multilingue de traductologie et traduction Translationes, éditée par le Centre de recherche ISTTRAROM-TRANSLATIONES, et du colloque international (En) Jeux esthétiques de la traduction. Éthique(s), technique et pratique traductionnelles, organisé par la Chaire de langues romanes et l'Association ISTTRAROM-TRANSLATIONES. Les nombreuses responsabilités que Jean-René Ladmiral assume sur le plan institutionnel donnent la mesure de la reconnaissance internationale dont jouit son savoir et son savoir-faire dans le domaine de la traduction, de la traductologie et de la médiation culturelle.

En évoquant sa prestigieuse carrière, nous avons tenu à souligner le rôle capital que Jean-René Ladmiral a joué dans la mise en place et le développement de l'enseignement de la traduction, la formation des traducteurs et la réflexion sur la traduction, rôle dont, en qualité de directeur de plusieurs centres de recherches, il a assuré le suivi et le rayonnement ; à le remercier pour toute son action, pour son dévouement inconditionnel et sans relâche à la cause de la traductologie, de la philosophie et de la linguistique. Dans la remise du titre de Docteur Honoris Causa de notre Alma Mater, il faut voir l'expression de l'hommage que nous avons le grand honneur de lui rendre pour l'ensemble de son œuvre, tout en appelant de nos vœux les plus chers une collaboration future riche et suivie. 


\section{Références bibliographiques}

Ladmiral, Jean-René. Traduire : théorèmes pour la traduction. Paris : Payot, [1979], 2002.

Ladmiral, Jean-René. "Les quatre âges de la traductologie - Réflexions sur une diachronie de la théorie de la traduction ». In : L'histoire et les théories de la traduction. Les actes (colloque de Genève : 3-5 octobre 1996). Berne \& Genève : ASTTI \& ETI, $1997:$ 11-42.

Ladmiral, Jean-René. " Dichotomies traductologiques ». In : La Linguistique, vol. 40, fas. (1) (2004) : 25-49.

Ladmiral, Jean-René et Edmond Marc Lipiansky, La communication interculturelle. Paris : Armand Colin, [1989] 1995.

Makaryeva, Anna. "L'impact des idées de Jean-René Ladmiral dans mon activité professionnelle et dans celle de mes collègues ». In: Transversalités; $\mathrm{n}^{\circ} 92$ (2004) : 103 .

Nègre, Fabien. La communication interculturelle chez Ladmiral et Lipiansky. In : Quaderni 22 (Hiver 1994) : 183. 